\title{
Une communauté de pratique en ligne à propos de l'enseignement de la compréhension de lecture au primaire : analyse des interactions et des niveaux de participation
}

Auteur(s)

Amélie Desmeules, Université Laval, Canada, amelie.desmeules@fse.ulaval.ca

Christine Hamel, Université Laval, Canada,

Christine.Hamel@fse.ulaval.ca

Marie-Catherine St-Pierre, Université Laval, Canada, marie-catherine.st-pierre@rea.ulaval.ca

Chantal Desmarais, Université Laval, Canada, chantal.desmarais@rea.ulaval.ca 


\title{
REVUE HYBRIDE DE L'ÉDUCATION
}

\begin{abstract}
Résumé
Afin d'assurer le développement professionnel d'enseignants quant à l'enseignement des habiletés langagières réceptives, socle de l'apprentissage de compréhension de lecture, cette recherche-action visait à mettre en place et à documenter une communauté de pratique $(\mathrm{CoP})$ en ligne. L'évolution des interactions dans la CoP a été analysée et des profils de participation ont été dégagés. Les résultats démontrent que les interactions de la deuxième phase de la CoP contribuent davantage à l'avancement du discours collectif et à la pérennité du modèle que celles de la première. Des recommandations sont émises quant à la mise en place des CoP et leur pérennisation.
\end{abstract}

Mots-clés: enseignement au primaire, compréhension de lecture, communauté de pratique, développement professionnel, interactions 


\section{6}

\section{REVUE HYBRIDE DE L'ÉDUCATION}

\section{Problématique}

\section{L'enseignement de la compréhension de la lecture}

Le langage sert de socle au développement de la compétence à lire au primaire, celle-ci étant un déterminant pour la persévérance et la réussite scolaires des élèves (Catts, Fey, Tomblin et Zhang, 2002 ; Hammer et al., 2017 ; Hattie, 2009 ; National Institute of Child Health and Human Development [NICHD], Early Child Care Research Network, 2005 ; Scarborough, 2001 ; Storch et Whitehurst, 2002). Le développement de la compétence à lire est particulièrement complexe puisqu'elle requiert à la fois un vocabulaire riche et diversifié (Braze, Tabor, Shankweiler et Mencl, 2007 ; Schatschneider, Fletcher, Francis, Carlson et Foorman, 2004 ; Verhoeven et Van Leeuwe, 2008) et l'habileté à créer une représentation du sens d'un texte (Cain et Oakhill, 2008). Enseigner à lire constitue donc un défi pour les enseignants et enseignantes qui doivent avoir recours à une variété de stratégies pour que les élèves développent leur compétence de façon optimale. Or, les enseignants et enseignantes du primaire se sentent, quant à eux, bien peu outillés pour soutenir le développement de la compréhension de lecture des élèves, plus particulièrement leurs habiletés inférentielles pour lesquelles les directives sont plutôt larges dans le curriculum d'études au Québec (MELS, 2006). Dans une étude pilote, $46 \%$ des enseignants et enseignantes questionnés précisaient d'ailleurs qu'ils considéraient stimuler les habiletés inférentielles chez leurs élèves de façon optimale et $54 \%$ ne le pensaient pas ou ne savaient pas s'ils le faisaient (St-Pierre et al., 2015). Ces données sont préoccupantes puisque les enseignants et enseignantes doivent appréhender avec justesse les concepts quant à la lecture et en posséder une connaissance spécialisée afin de les enseigner avec succès (Piasta et al. 2009).

\section{Soutenir le développement professionnel par la mise en place d'une communauté de pratique}

Malgré l'importance reconnue de la compétence à lire et les besoins de développement professionnel des enseignants et enseignantes à cet effet, les études qui portent précisément sur leur développement professionnel à l'égard de l'enseignement de la compréhension de lecture sont peu nombreuses (St-Pierre et al., 2015). Pour assurer le développement de leurs pratiques et de leurs connaissances, il nous est apparu pertinent de mettre en place un dispositif de développement professionnel en regard des habiletés langagières réceptives (combinant les inférences, le vocabulaire et la morphosyntaxe) qui tient compte des connaissances scientifiques actuelles (Nation, Clarke, Marshall et Durand, 2004 ; Oakhill et Cain, 2004). Le dispositif en ligne déployé est basé sur le modèle théorique de la Communauté de pratique (CoP) (Wenger, 2005). $\mathrm{Ce}$ modèle répond aux critères d'efficacité du développement professionnel et il permet, par l'engagement et la participation de ces membres, le renouvellement des pratiques (Boutin et Hamel, 2017). En 


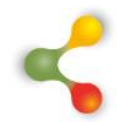

\section{REVUE HYBRIDE DE L'ÉDUCATION}

effet, plusieurs recherches ont documenté la mise en œuvre de communautés de pratique intentionnelles d'enseignants et enseignantes et ont mis de l'avant des résultats qui permettent de répondre à plusieurs critères d'efficacité du développement professionnel (Boutin et Hamel, 2017). Ces dispositifs de développement professionnel seraient efficaces parce qu'ils ont le souci d'offrir un accompagnement prolongé (Blank et De las Alas, 2009 ; Desimone, 2009 ; Wood et Stanulis, 2009), soit par les pairs ou par des professionnels (Gaudreau et Nadeau, 2015), qui prend ses assises de la pratique réelle et des enjeux authentiques qui amènent les apprenants à se questionner en vue de trouver des pistes de régulation (Bissonnette et Richard, 2010 ; Lussi Borer et Muller, 2017 ; Wood et Stanulis, 2009).

\section{Le déploiement et la pérennité des dispositifs de développement professionnel}

Bien qu'il soit reconnu que le modèle de communauté de pratique répond bien aux critères d'efficacité du développement professionnel, peu d'études documentent leur déploiement ainsi que leur pérennité (Bourhis et Tremblay, 2004 ; Dameron et Josserand, 2007 ; Dubé, Bourhis et Jacob, 2005 ; McDermott, 2000 ; Wenger, McDermott et Snyder, 2002). Dans leurs travaux, Bourhis et ses collaborateurs se sont penchés sur la question, notamment au regard des facteurs organisationnels des communautés de pratiques virtuelles (CoPV) dont découlait le succès de leur déploiement à long terme, prenant appui sur leur niveau de vitalité, entre autres. Ils ont mis en lumière le fait que plus l'environnement organisationnel de la CoPV est facilitant et plus les sujets abordés sont pertinents pour ses membres, plus la vitalité de la CoPV était élevée. De même, lorsque les membres de la gouvernance de la CoPV sont très impliqués dans le discours et l'avancement de la CoPV, la vitalité de celleci est de plus haut niveau (Bourhis et Tremblay, 2004 ; Dubé, Bourhis et Jacob, 2005). Par ailleurs, comme l'ont identifié Boutin et Hamel dans une étude menée en 2017 ,à l'exception du coordonnateur et animateur qui a mis en place et coordonne la communauté de pratique, les membres font rarement des activités contribuant à l'avancement du discours collectif et, par le fait même, à la pérennité de la communauté dans une visée de déploiement. Selon les auteurs, "cela pourrait signifier que les enseignants [...] n'ont pas endossé la responsabilité d'amener le discours plus loin en termes de planification et de suivi, laissant cette responsabilité à la professionnelle » (Boutin et Hamel, 2017, p.17). Ainsi, il serait pertinent de documenter les interactions à l'intérieur d'un dispositif de communauté de pratique afin de constater si des profils de participation peuvent être dégagés au sein des membres ne faisant pas partie de l'équipe de coordination initiale et, le cas échéant, s'ils peuvent avoir un impact sur la responsabilité collective (c.-à-d. la responsabilité du succès de la communauté répartie entre tous ses membres et non seulement ceux de l'équipe de coordination (Scardamalia, 2002) et la pérennité du modèle mis 


\section{$\&$}

\section{REVUE HYBRIDE DE L'ÉDUCATION}

en place pour soutenir le développement de pratiques chez les enseignants et enseignantes.

\section{Cadre théorique}

La CoP qui a été mise en place dans le cadre de notre projet s'inscrit dans la théorie des communautés de pratique proposée par Wenger (2005). Selon cette théorie, l'apprentissage est conçu sous l'angle de la participation sociale, soit " une collaboration active aux pratiques d'une communauté sociale » et une " construction d'identités en lien avec celles-ci » (Wenger, 2005, p.2). La pratique est alors conçue comme une construction de sens en tant qu'expérience de la vie quotidienne qui est négociée par la participation et la réification. La participation renvoie à l'action et à la mise en relation avec d'autres personnes où s'observe une reconnaissance mutuelle tandis que la réification renvoie au processus qui donne forme à l'expérience, elle est au cœur de chaque pratique et mobilise l'énergie collective afin de créer un répertoire collectif. La négociation de sens est donc liée à la constitution de communauté pratique puisque la pratique est la propriété d'une communauté. Ainsi, elle repose sur trois dimensions, soit l'entreprise commune, l'engagement mutuel et le répertoire partagé.

La négociation de sens permet aux participants d'apprendre au sein des interactions dans la communauté (Wenger, 2005). Celles-ci prennent dès lors une grande importance puisque le discours collectif qui y prend place leur permet de coconstruire leurs connaissances. Plusieurs auteurs se sont d'ailleurs intéressés à cet aspect dans le contexte du discours écrit, à l'intérieur de forum collaboratif, par exemple (Bereiter et Scardamalia, 1987 ; Boutin, Hamel et Laferrière, 2016 ; Garrison, Anderson et Archer, 2000 ; Murphy, 2004 ; Scardamalia et Bereiter, 2003 ). Murphy (2004), se basant sur les travaux de Garrison et ses collaborateurs (2000), met de l'avant que plus le discours est partagé, plus la coconstruction au sein de la communauté est importante. Pour ce faire, les interventions au sein du discours ne doivent pas être individuelles, mais plutôt prendre en compte les perspectives des autres, les confronter et les affiner (Murphy, 2004).

L'évolution du déploiement d'une CoP se réalise en cinq stades de développement selon Wenger et al. (2002), soit le potentiel, l'unification, la maturité, le momentum et la transformation (ou la dissolution). Autrement dit, un réseau informel de personnes où s'observe une reconnaissance mutuelle s'établit à l'étape du potentiel, lequel se formalise et se met en place à l'étape d'unification et gagne en maturité avec la participation et l'avancement du discours collectif. À cette étape de la maturité, les membres clarifient l'objet et les rôles de la CoP et de nouvelles connaissances seront développées par la négociation de sens (Langelier, 2005). À l'étape du momentum, la CoP, faisant face à des changements inévitables du point de vue de l'organisation, doit maintenir sa vitalité sinon 


\section{$\&$}

\section{REVUE HYBRIDE DE L'ÉDUCATION}

se transformer ou éventuellement, se dissoudre. Le modèle que proposent Wenger et al. (2002) est linéaire, mais la durée de chaque étape est variable. Le modèle de Snoeck et Tilman (2004) propose plutôt que cette évolution est itérative et donc que des allers-retours sont possibles, voire nécessaires, entre les étapes.

Par ailleurs, afin d'analyser les interactions, le modèle de participation à la CoP proposé par Wenger, McDermott et Snyder (2002) a été utilisé. Comme présenté dans la Figure 1, il y a trois niveaux de participation à la communauté à l'intérieur desquels les membres vont exercer différentes fonctions. Ainsi, dans le niveau central, les membres sont les plus entrepreneurs et sont essentiels afin de faire avancer et évoluer la communauté. Ils participent activement, ils soulèvent des questionnements et des sujets pertinents pour l'ensemble de la communauté et ils vont également débattre pour faire avancer les idées (Wenger, McDermott et Snyder, 2002). C'est habituellement à ce niveau que se retrouve l'équipe de coordination.

Au niveau suivant, les membres seront actifs à l'intérieur de la communauté. Ils participent régulièrement aux rencontres et prennent part aux interactions. Cela dit, ils ne seront pas aussi présents et entrepreneurs que les membres du niveau central. II y a généralement un peu plus de participants à ce niveau (Wenger, McDermott et Snyder, 2002).

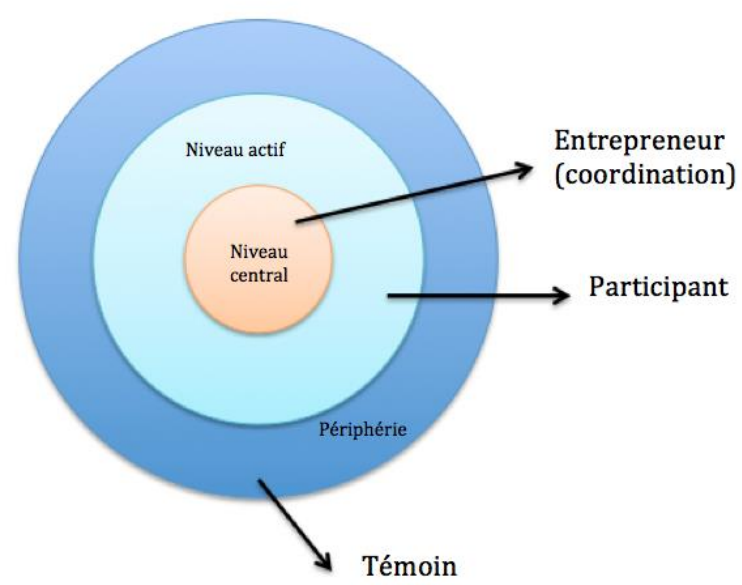

Figure 1. Le modèle de participation à la CoP (Wenger, McDermott et Snyder, 2002, p.57) 


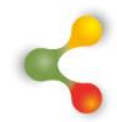

\section{REVUE HYBRIDE DE L'ÉDUCATION}

Vient ensuite le niveau périphérique où les membres ne participent qu'à quelques reprises, et ce, à titre de témoins, observant les interactions entre les membres entrepreneurs et les membres participants. II semble que deux raisons mènent les membres d'une communauté de pratique à ne pas interagir, soit le sentiment d'incompétence et d'imposture ou encore le manque de temps disponible pour s'investir (Wenger, McDermott et Snyder, 2002). II importe de mentionner que la participation des membres est en mouvance continue au sein d'une communauté de pratique. Ainsi, les membres peuvent passer d'un niveau à l'autre et varier leur participation en fonction de leur intérêt et de leur disponibilité au sein de ce collectif.

\section{Objectif de recherche}

Notre objectif de recherche est donc de documenter l'évolution des interactions à l'intérieure de la CoP au fil des deux phases du projet afin de dégager des profils de participation. Comment les interactions à l'intérieur de la CoP ont-elles évolué au fil des deux phases du projet et quels profils de participation se dégagent à l'intérieur de la CoP ?

\section{Méthodologie}

\section{Mise en contexte}

Dans le cadre d'un projet de recherche-action subventionné par le programme d'actions concertées (Fonds de recherche du Québec Société et Culture), nous avons mis en place un dispositif de développement professionnel qui avait pour objectif de soutenir des enseignantes dans l'enseignement de la compréhension de lecture en favorisant le développement et l'utilisation des habiletés langagières réceptives chez leurs élèves. Le déroulement du dispositif s'est étendu sur deux phases. La première phase s'est déroulée du mois de janvier au mois de juin 2015 à raison de cinq rencontres de 90 minutes, dont une de rétroaction individuelle. Elle était animée par la chercheuse principale du projet de recherche qui était assistée par une étudiante à la maitrise en psychopédagogie et une orthophoniste.

Les analyses préliminaires de cette première phase ont permis d'orienter les activités de la deuxième phase, qui elle, s'est déroulée entre le mois d'octobre 2015 et le mois de mai 2016 à raison de huit rencontres dont deux de rétroaction individuelle. L'analyse préliminaire des interactions à la suite de la phase $1 \mathrm{a}$ mené à la recommandation de confier l'animation à une personne tierce, qui tout en étant formée dans le domaine de l'éducation, était moins impliquée dans la conceptualisation de la recherche afin de favoriser les interactions entre les participants. L'animation a ainsi été confiée à l'étudiante à la maitrise qui était assistée par l'orthophoniste. Lors des rencontres des deux phases, une structure similaire était proposée aux participantes. Elles devaient d'abord préparer et filmer une activité de compréhension de lecture ciblant le développement 


\section{$\&$}

\section{REVUE HYBRIDE DE L'ÉDUCATION}

des habiletés inférentielles en classe. Elles faisaient ensuite un retour sur l'activité vécue en partageant leur expérience avec les autres participantes dans la première partie de la rencontre en ligne. À la suite de ce partage, elles échangeaient sur un thème ciblé en lien avec leurs expériences (par exemple : notions théoriques, stratégies pédagogiques). Finalement, elles se remettaient en action afin de planifier et de vivre une nouvelle activité de compréhension de lecture ciblant le développement des habiletés inférentielles pour la rencontre suivante.

\section{Recrutement}

Les participantes étaient des enseignantes de l'ordre primaire participant à l'École en Réseau (ÉER). Ce modèle et les enseignants et enseignantes qui y participent ont été choisis puisqu'ils répondent à certains critères d'inclusion, soit d'enseigner dans des milieux ruraux et défavorisés. II s'agit donc d'un échantillonnage non probabiliste. Le recrutement a été fait sous forme d'envois électroniques à partir de la liste de diffusion de l'ÉER. II s'est déroulé entre les mois de septembre 2014 et de janvier 2015 lors de la première phase de la communauté de pratique et celui pour la deuxième phase s'est déroulé entre le mois de juin et le mois de septembre 2015.

\section{Participants}

L'échantillon de l'étude est constitué de dix enseignantes du primaire $(n=10)$, soit six participantes pour la phase 1 et six à la phase 2 , dont deux ont également participé à la phase 1. II est à noter qu'au départ, sept enseignantes se sont inscrites à la première phase de la CoP, mais l'une a eu un changement de tâche important en cours de projet ce qui a entrainé son abandon. De même, huit enseignantes se sont inscrites à la deuxième phase de la CoP, mais deux de celles-ci ont abandonné le projet après deux rencontres. Ces trois participantes ont été exclues des analyses. Afin de conserver l'anonymat des participantes, celles-ci seront désignées de la façon suivante dans cet article : E1, E2, E3, E4, E5, E6, $E 10, E 11, E 12, E 13$. Les participantes $E 1$ et E5 sont les deux seules qui ont participé aux deux phases de la communauté de pratique. Le tableau 1 représente la répartition des participantes en fonction des caractéristiques sociodémographiques. Les participantes sont des femmes, âgées entre 25 à 59 ans et ayant en moyenne 20 années d'expérience d'enseignement. Les cycles représentés sont les premier, deuxième et troisième cycles. Les participantes proviennent de différentes régions à travers le Québec réparties dans six commissions scolaires. Les moyennes des indices du Seuil de Faible Revenu (ISFR) et du milieu socioéconomique (MSE) sont respectivement de 4,2 sur 10 et de 8,2 sur 10 . 


\section{REVUE HYBRIDE DE L'ÉDUCATION}

\section{Tableau 1}

Répartition des participantes selon les caractéristiques sociodémographiques $(n=10)$

\begin{tabular}{lc}
\hline \multicolumn{1}{c}{ Caractéristiques sociodémographiques } & Participants $\mathrm{n}=10$ \\
\hline Âge des participants n (\%) & $1(10)$ \\
$25-29$ & $2(20)$ \\
$35-39$ & $2(20)$ \\
$40-44$ & $2(20)$ \\
$45-49$ & $2(20)$ \\
$50-54$ & $1(10)$ \\
$55-59$ & $10(100)$ \\
Sexe & $19,9(10,84)$ \\
Féminin n (\%) & \\
Expérience d'enseignement (an) M (ET) & $6(60)$ \\
Niveau d'enseignement & $3(30)$ \\
Premier cycle & $1(10)$ \\
Deuxième cycle & \\
Multi cycle (2 et 3) & $1(10)$ \\
Régions n (\%) & $3(30)$ \\
Chaudière-Appalaches & $2(20)$ \\
Laurentides & $1(10)$ \\
Saguenay-Lac-Saint-Jean & $1(10)$ \\
Gaspésie & $2(20)$ \\
Bas-Saint-Laurent & \\
Estrie & $8,2(1,81)$ \\
Défavorisation des milieux & \\
Indice du milieu socio-économique (rang & \\
Indice du Seuil de Faible Revenu (rang & \\
décile) M (ET) & \\
\hline & \\
\hline
\end{tabular}

\section{Analyse des interactions}

Afin d'analyser les interactions à l'intérieur des rencontres de la CoP, les enregistrements de toutes les rencontres ont été retranscrits. L'analyse de ces verbatims a mené à un codage d'interventions à l'intérieur 


\section{6}

\section{REVUE HYBRIDE DE L'ÉDUCATION}

des enregistrements de chacune des rencontres. Plus précisément, nous avons procédé à l'écoute de chacun des enregistrements des rencontres puis avons codé dans des tableaux toutes les interventions ou prises de parole qui ont eu lieu à l'intérieur d'une même rencontre. Les analyses ont permis de répertorier : 1) le nombre d'interventions par participantes, 2) le temps d'interventions total de chaque participante de même que 3) la nature des interventions de celles-ci.

Par ailleurs, le codage de la nature des interventions est émergent. Nous avons élaboré les codes sur la base de la fonction de l'intervention et de la relation sémantique entre les concepts de cette intervention (Krippendorff, 2013). À titre d'exemple, si l'intervention d'une participante consistait à poser une question, alors elle était codée "Pose une question ». Le codage de l'ensemble des interventions qui ont pris place au cours des huit rencontres a été réalisé par un seul codeur afin d'assurer une fidélité du codage intrajuge (Miles et Huberman, 2003). Toutefois, afin d'assurer la validité des codes émergents qui ont été définis, un accord interjuge a été complété entre deux membres de l'équipe de recherche. Cet exercice nous a permis de préciser les définitions des codes, car au premier interjuge, l'alpha d'accord pour deux rencontres codées était de $60 \%$ et au deuxième interjuge, il est passé à $73 \%$ avec une troisième rencontre codée. Comme il s'agit de codage émergent, un alpha d'accord élevé est plus difficile à obtenir et le score obtenu est suffisant pour prétendre à une validité du construit (Krippendorff, 2013). Par la suite, nous avons regroupé les codes obtenus en quatre niveaux d'expertise de prise en compte des autres membres dans la communication basés sur les travaux de BoixMansilla et Gardner (1998), soit "naïf », "novice », " apprenti » et " expert ". Cette catégorisation nous permet de qualifier la nature des interventions réalisées par les participantes dans la CoP, et ce, au regard de leur niveau d'expertise dans l'avancement du discours collectif. Rappelons que comme l'apprentissage dans la CoP se concrétise par la négociation de sens (Wenger, 2005), il est effectivement important de considérer la prise en compte des autres participants à l'intérieur des interventions identifiées pour mieux comprendre leur apport au discours collectif. Le tableau 2 présente les libellés et descriptions des codes émergents.

\section{Résultats}

\section{L'évolution des interactions au fil des deux phases}

Afin de répondre à nos questions de recherche, soit de constater l'évolution des interactions à l'intérieur de la CoP en vue d'établir des profils de participants, nous avons d'abord comparé le nombre d'interventions de chaque participante de même que leur longueur. Les tableaux 3 et 4 présentent les données obtenues pour les cinq premières rencontres de la CoP des deux phases (1 et 2). Nous avons choisi de comparer seulement les cinq premières pour que l'échantillonnage de rencontres soit équivalent, 


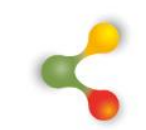

\section{REVUE HYBRIDE DE L'ÉDUCATION}

ce qui n'était pas le cas au départ (phase $1 n=5$ rencontres et phase $2 n=8$ rencontres).

\section{Tableau 2}

Libellés et descriptions des codes émergents 


\section{REVUE HYBRIDE DE L'ÉDUCATION}

\begin{tabular}{|c|c|c|}
\hline $\begin{array}{c}\text { Niveaux } \\
\text { d'expertise } \\
\text { de prise en } \\
\text { compte } \\
\text { des autres } \\
\text { membres } \\
\text { dans le } \\
\text { discours }\end{array}$ & $\begin{array}{l}\text { Libellé du } \\
\text { code }\end{array}$ & Description du code \\
\hline Naïf & $\begin{array}{l}\text { Donne une } \\
\text { réponse }\end{array}$ & $\begin{array}{l}\text { Une intervention égocentrée, } \\
\text { plutôt courte, qui survient à la } \\
\text { suite d'une question et qui } \\
\text { exprime une seule idée. Les } \\
\text { réponses ne reposent pas sur } \\
\text { une analyse ou une réflexion } \\
\text { élaborée. Les réponses ne } \\
\text { servent pas à faire avancer la } \\
\text { discussion avec les autres. Ex. : } \\
\text { «J'enseigne dans une classe de } \\
6^{\mathrm{e}} \text { année. J'ai bien compris. » }\end{array}$ \\
\hline \multirow[b]{2}{*}{ Novice } & $\begin{array}{l}\text { Apporte } \\
\text { une } \\
\text { précision }\end{array}$ & $\begin{array}{l}\text { Une intervention qui permet } \\
\text { d'apporter une précision sur une } \\
\text { autre intervention (d'autrui ou de } \\
\text { soi). Cette intervention n'amène } \\
\text { pas de nouvelles idées ou de } \\
\text { nouveaux sujets, mais prend en } \\
\text { considération la compréhension } \\
\text { des autres membres. Ex. : « Je } \\
\text { préciserais par rapport à ça... » }\end{array}$ \\
\hline & $\begin{array}{l}\text { Donne une } \\
\text { information } \\
\text { nouvelle }\end{array}$ & $\begin{array}{l}\text { Une intervention courte ou longue } \\
\text { pour donner une information sur } \\
\text { un sujet ou un aspect précis et } \\
\text { nouveau pour mieux permettre la } \\
\text { compréhension des autres } \\
\text { membres. Le sujet n'a pas été } \\
\text { abordé précédemment et } \\
\text { l'intervention ne suit pas une } \\
\text { question. Les informations } \\
\text { données peuvent faire avancer la } \\
\text { discussion, mais ce n'est pas leur } \\
\text { intention première. Ex. : « Je ne } \\
\text { l'avais pas dit, mais j'ai un groupe } \\
\text { difficile. » }\end{array}$ \\
\hline Apprenti & $\begin{array}{l}\text { Pose une } \\
\text { question }\end{array}$ & $\begin{array}{l}\text { Une intervention plutôt courte qui } \\
\text { peut être une question ouverte ou } \\
\text { fermée. Les autres membres sont } \\
\text { pris en considération. Ex. : } \\
\text { "Peux-tu répéter? ", " Comment } \\
\text { avez-vous trouvé l'activité? » }\end{array}$ \\
\hline
\end{tabular}




\section{REVUE HYBRIDE DE L'ÉDUCATION}

\begin{tabular}{|c|c|}
\hline $\begin{array}{l}\text { Exprime } \\
\text { une idée }\end{array}$ & $\begin{array}{l}\text { Une intervention assez longue } \\
\text { qui survient à la suite d'une } \\
\text { consigne donnée ou d'une } \\
\text { question posée. Il s'agit d'un } \\
\text { ensemble d'idées portant sur un } \\
\text { sujet précis qui est en lien direct } \\
\text { avec la consigne donnée ou la } \\
\text { question posée. } \\
\text { L'idée repose sur une analyse qui } \\
\text { est expliquée de sorte que les } \\
\text { autres membres comprennent } \\
\text { bien ce dont il est question. Ils } \\
\text { sont donc pris en considération. } \\
\text { Ex. : Raconter le déroulement } \\
\text { d'une activité, donner une } \\
\text { définition personnelle d'un } \\
\text { concept. }\end{array}$ \\
\hline $\begin{array}{l}\text { Donne son } \\
\text { opinion }\end{array}$ & $\begin{array}{l}\text { Une intervention qui sert à } \\
\text { partager son opinion avec les } \\
\text { autres sans toutefois appeler à } \\
\text { une rétroaction de leur part. Ex. : } \\
\text { « Je suis d'accord pour qu'on } \\
\text { fonctionne de cette façon », " Ça } \\
\text { me va ». }\end{array}$ \\
\hline $\begin{array}{l}\text { Élabore } \\
\text { sur une } \\
\text { réponse } \\
\text { donnée }\end{array}$ & $\begin{array}{l}\text { Une intervention à la suite d'une } \\
\text { autre intervention et qui prend } \\
\text { appui sur celle-ci. Ces } \\
\text { interventions servent à faire } \\
\text { avancer la discussion avec les } \\
\text { autres qui sont interpelés. } \\
\text { L'intervention peut amener une } \\
\text { rétroaction par rapport à la } \\
\text { compréhension ou non du propos } \\
\text { suite à l'intervention d'un autre } \\
\text { membre. Ex. : « Je reviens sur ce } \\
\text { que Gisèle vient de dire »; «Ce } \\
\text { que tu viens de dire ça me fait } \\
\text { penser à ça ». }\end{array}$ \\
\hline $\begin{array}{l}\text { Reformule } \\
\text { un propos }\end{array}$ & $\begin{array}{l}\text { Une intervention pour reformuler } \\
\text { le propos d'une autre } \\
\text { intervention. L'intervention relève } \\
\text { d'une sensibilité par rapport aux } \\
\text { interventions des autres membres } \\
\text { et à ses propres interventions et } \\
\text { leurs effets sur les autres } \\
\text { membres. L'intervention peut } \\
\text { amener une rétroaction par } \\
\text { rapport à la compréhension ou }\end{array}$ \\
\hline
\end{tabular}




\section{REVUE HYBRIDE DE L'ÉDUCATION}

non du propos à la suite de

l'intervention d'un autre membre.

Ex. : « Si je reformule ce que tu

viens de dire... »

\section{Tableau 3}

Comparaison de l'évolution du temps d'intervention au fil des cinq premières rencontres de la CoP phases 1 et 2 par participantes

\begin{tabular}{|c|c|c|c|c|c|}
\hline Participantes & CoP \#1 & CoP \#2 & CoP \#3 & CoP \#4 & CoP \#5 \\
\hline E1 (Phase 1) & 00:03 & 00:06 & $\begin{array}{c}00: 11: 1 \\
5\end{array}$ & $\begin{array}{c}00: 07: 3 \\
5\end{array}$ & $\begin{array}{c}00: 05: 0 \\
0\end{array}$ \\
\hline E2 (Phase 1) & 00:02 & 00:07 & $\begin{array}{c}00: 03: 0 \\
0\end{array}$ & $\begin{array}{c}00: 06: 2 \\
0\end{array}$ & $\begin{array}{c}00: 00: 0 \\
0\end{array}$ \\
\hline E3 (Phase 1) & 00:00 & 00:00 & $\begin{array}{c}00: 04: 4 \\
5\end{array}$ & $\begin{array}{c}00: 03: 0 \\
5\end{array}$ & $\begin{array}{c}00: 06: 2 \\
0\end{array}$ \\
\hline E4 (Phase 1) & 00:02 & 00:09 & $\begin{array}{c}00: 04: 0 \\
0\end{array}$ & $\begin{array}{c}00: 04: 1 \\
0\end{array}$ & $\begin{array}{c}00: 09: 0 \\
0\end{array}$ \\
\hline E5 (Phase 1) & $00: 00$ & $\begin{array}{c}00: 00: 0 \\
0\end{array}$ & $\begin{array}{c}00: 04: 3 \\
0\end{array}$ & $\begin{array}{c}00: 05: 4 \\
0\end{array}$ & $\begin{array}{c}00: 09: 2 \\
5\end{array}$ \\
\hline E6 (Phase 1) & $\begin{array}{c}00: 04: 5 \\
5 \\
00 \cdot 05 \cdot 5\end{array}$ & $\begin{array}{c}00: 00: 0 \\
0 \\
00: 06: 1\end{array}$ & $\begin{array}{c}00: 06: 1 \\
0 \\
00: 16: 1\end{array}$ & $\begin{array}{c}00: 00: 0 \\
0 \\
00: 12 \cdot 0\end{array}$ & $\begin{array}{c}00: 00: 0 \\
0 \\
00 \cdot 00 \cdot 0\end{array}$ \\
\hline E1 (Phase 2) & $\begin{array}{c}5 \\
00: 10: 0\end{array}$ & $\begin{array}{c}1 \\
00: 05: 0\end{array}$ & $\begin{array}{c}5 \\
00: 12: 1\end{array}$ & $\begin{array}{c}2 \\
00: 00: 0\end{array}$ & $\begin{array}{c}0 \\
00: 12: 1\end{array}$ \\
\hline E5 (Phase 2) & $\begin{array}{c}6 \\
00: 02: 0\end{array}$ & $\begin{array}{c}8 \\
00: 00: 0\end{array}$ & $\begin{array}{c}2 \\
00: 10: 2\end{array}$ & $\begin{array}{c}0 \\
00: 04: 3\end{array}$ & $\begin{array}{c}7 \\
00: 13: 1\end{array}$ \\
\hline E10 (Phase 2) & $\begin{array}{c}9 \\
00: 06: 3\end{array}$ & $\begin{array}{c}0 \\
00: 06: 1\end{array}$ & 4 & $\begin{array}{c}4 \\
00: 00: 0\end{array}$ & $\begin{array}{c}7 \\
00: 13: 4\end{array}$ \\
\hline E11 (Phase 2) & $\begin{array}{c}2 \\
00: 04: 1\end{array}$ & $\begin{array}{c}5 \\
00: 03: 3\end{array}$ & $\begin{array}{c}00: 16 \\
00: 17: 4\end{array}$ & $\begin{array}{c}0 \\
00: 08: 4\end{array}$ & $\begin{array}{c}7 \\
00: 12: 3\end{array}$ \\
\hline $\begin{array}{l}\text { E12 (Phase 2) } \\
\text { E13 (Phase 2) }\end{array}$ & $\begin{array}{c}1 \\
00: 00: 0 \\
0 \\
\end{array}$ & $\begin{array}{c}1 \\
00: 00: 4 \\
9 \\
\end{array}$ & $\begin{array}{c}0 \\
00: 13\end{array}$ & $\begin{array}{c}0 \\
00: 06: 1 \\
5 \\
\end{array}$ & $\begin{array}{c}0 \\
00: 20: 0 \\
5 \\
\end{array}$ \\
\hline
\end{tabular}

Dans le tableau 3, le temps d'intervention des participantes de la phase 2 est considérablement plus important que le temps d'interventions des participantes de la phase 1 , et ce, pour les cinq premières rencontres des deux phases. II y a seulement à la deuxième rencontre de la deuxième phase que le temps de parole des participantes a été un peu moins élevé que celle de la phase 1. À cet effet, il importe de mentionner que la chercheuse principale, qui animait la CoP lors de la première phase, a présenté aux participantes (caméra et micro ouverts) des contenus théoriques portant sur les stratégies de rétroaction et de modelage langagier pendant environ 30 minutes lors de cette rencontre. 


\section{REVUE HYBRIDE DE L'ÉDUCATION}

\section{Tableau 4}

Comparaison de l'évolution du nombre d'interventions au fil des cinq premières rencontres de la CoP phase 1 et 2 par participantes

\begin{tabular}{lccccc}
\hline \multicolumn{1}{c}{ Participantes } & CoP & CoP & CoP & CoP & CoP \\
& $\# 1$ & $\# 2$ & $\# 3$ & $\# 4$ & $\# 5$ \\
\hline E1 (Phase 1) & 4 & 8 & 8 & 7 & 11 \\
E2 (Phase 1) & 5 & 6 & 5 & 10 & 0 \\
E 3 (Phase 1) & 5 & 0 & 5 & 4 & 9 \\
E4 (Phase 1) & 7 & 10 & 6 & 7 & 12 \\
E 5 (Phase 1) & 0 & 0 & 4 & 6 & 10 \\
E 6 (Phase 1) & 8 & 4 & 7 & 0 & 0 \\
E1 (Phase 2) & 10 & 5 & 13 & 12 & 3 \\
E5 (Phase 2) & 23 & 2 & 20 & 0 & 8 \\
E10 (Phase 2) & 12 & 1 & 13 & 10 & 43 \\
E11 (Phase 2) & 18 & 8 & 52 & 0 & 34 \\
E12 (Phase 2) & 14 & 3 & 25 & 14 & 30 \\
E13 (Phase 2) & 0 & 2 & 30 & 10 & 39 \\
\hline
\end{tabular}

Par ailleurs, dans le tableau 4, nous pouvons également constater qu'au-delà du temps d'intervention, le nombre d'interventions des participantes de la phase 2 est considérablement plus important que le nombre d'interventions des participantes de la phase 1, sauf à la deuxième rencontre. Encore une fois, il s'agit de la rencontre où la chercheuse principale a participé aux interactions. De plus, les participantes E1 et E2 qui ont participé aux deux phases de la CoP ont également augmenté la fréquence et la longueur de leurs interventions d'une phase à l'autre. Elles sont donc de bons indicateurs de l'évolution des interactions entre les deux phases.

Toujours afin de répondre à nos questions de recherche, nous avons également comparé la nature des interventions de chaque participante de même que leur niveau d'expertise de prise en compte des autres dans l'avancement du discours collectif (Boix-Mansilla et Gardner, 1998). Les tableaux 5 et 6 présentent les données obtenues pour les rencontres de la CoP des deux phases (phase $1 n=5$ rencontres et phase $2 \mathrm{n}=8$ rencontres). 


\section{REVUE HYBRIDE DE L'ÉDUCATION}

\section{Tableau 5}

Niveau d'expertise des interventions des participantes de la phase 1 réalisées pendant les cinq rencontres de la communauté de pratique $(n=160)$

\begin{tabular}{lcccc}
\hline Participantes & $\begin{array}{c}\text { Naïf } \\
\mathrm{n}(\%)\end{array}$ & $\begin{array}{c}\text { Novice } \\
\mathrm{n}(\%)\end{array}$ & $\begin{array}{c}\text { Apprenti } \\
\mathrm{n}(\%)\end{array}$ & $\begin{array}{c}\text { Expert } \\
\mathrm{n}(\%)\end{array}$ \\
\hline E1 & $12(32,4)$ & $1(2,7)$ & $17(45,9)$ & $7(18,9)$ \\
E2 & $11(37,9)$ & $1(3,4)$ & $14(48,2)$ & $3(10,3)$ \\
E3 & $12(52,1)$ & $0(0)$ & $10(43,4)$ & $1(4,3)$ \\
E4 & $9(25,7)$ & $0(0)$ & $22(62,8)$ & $4(11,4)$ \\
E5 & $8(47,1)$ & $0(0)$ & $9(52,9)$ & $0(0)$ \\
E6 & $10(52,6)$ & $7(36,8)$ & $0(0)$ & $2(10,5)$ \\
\hline
\end{tabular}

Tableau 6

Niveau d'expertise des interventions des participantes de la phase 2 réalisées pendant les huit rencontres de la communauté de pratique $(n=582)$

\begin{tabular}{lcccc}
\hline Participantes & $\begin{array}{c}\text { Naïf } \\
\mathrm{n}(\%)\end{array}$ & $\begin{array}{c}\text { Novice } \\
\mathrm{n}(\%)\end{array}$ & $\begin{array}{c}\text { Apprenti } \\
\mathrm{n}(\%)\end{array}$ & $\begin{array}{c}\text { Expert } \\
\mathrm{n}(\%)\end{array}$ \\
\hline E1 & $15(22)$ & $3(4,4)$ & $42(61,7)$ & $8(11,7)$ \\
E5 & $43(34,4)$ & $5(4)$ & $66(52,8)$ & $11(8,8)$ \\
E10 & $22(25,8)$ & $7(8,2)$ & $51(60)$ & $5(5,8)$ \\
E11 & $31(23,3)$ & $9(6,7)$ & $80(79,3)$ & $13(9,7)$ \\
E12 & $13(14,9)$ & $11(12,6)$ & $55(63,2)$ & $8(9,1)$ \\
E13 & $31(36,9)$ & $9(10,7)$ & $42(50)$ & $2(2,3)$ \\
\hline
\end{tabular}

Certains constats peuvent être dégagés de ces deux tableaux. D'abord, le niveau d'expertise des interventions est beaucoup plus varié (et donc de natures plus variées) lors de la phase 2 que lors de la phase 1. Toutefois, dans les deux phases, la majorité des interventions faites sont de niveau apprenti. Par ailleurs, le nombre d'interventions de niveau expert est beaucoup plus important lors de la phase 2. De plus, toujours quant aux interventions de niveau expert, la participante $E 1$ se démarque des autres participantes tant lors de la première phase que lors de la deuxième phase. 


\section{$\&$}

\section{REVUE HYBRIDE DE L'ÉDUCATION}

En effet, parmi ses interventions totales, c'est elle qui a la plus grande proportion d'intervention de niveau expert. C'est donc la participante qui a le plus souvent élaboré sur les interventions des autres et reformulé sa compréhension des interventions des autres de sorte à donner des rétroactions à ses collègues et à faire avancer le discours collectif de la communauté de pratique. Par ailleurs, la participante E5 se démarque également quant à l'évolution considérable du niveau d'expertise de son discours, passant d'aucune intervention de niveau expert lors de la phase 1 à 11 interventions de ce niveau lors de la phase 2 .

\section{Les niveaux et profils de participation observés}

Dans un autre ordre d'idées, afin de répondre plus spécifiquement à notre deuxième question de recherche, nous avons observé les niveaux de participation parmi les rencontres de la première phase et de la deuxième phase de la communauté de pratique. Ces niveaux ont été observés à partir de la présence aux rencontres de la CoP et de l'investissement dans les activités proposées, soit par le nombre d'activités réalisées et le nombre d'activités déposées. En effet, il était demandé aux participantes de réaliser une activité après chaque rencontre, de la filmer et de déposer l'enregistrement sur la plateforme en ligne pour qu'il soit accessible aux autres participantes. Les tableaux 7 et 8 présentent ces données issues des deux phases.

\section{Tableau 7}

Présence aux rencontres de la CoP et participation aux activités proposées (phase 2)

\begin{tabular}{lccc}
\hline Participantes & $\begin{array}{c}\text { Présences aux } \\
\text { CoP } \\
(\mathrm{n}=8)\end{array}$ & $\begin{array}{c}\text { Activités } \\
\text { réalisées } \\
(\mathrm{n}=3)\end{array}$ & $\begin{array}{c}\text { Activités } \\
\text { déposées } \\
(\mathrm{n}=3)\end{array}$ \\
\hline Enseignante 1 & 8 & 4 & 4 \\
Enseignante 5 & 7 & 3 & 3 \\
Enseignante 10 & 7 & 2 & 2 \\
Enseignante 11 & 7 & 3 & 3 \\
Enseignante 12 & 8 & 3 & 3 \\
Enseignante 13 & 6 & 2 & 2 \\
\hline
\end{tabular}




\section{REVUE HYBRIDE DE L'ÉDUCATION}

\section{Tableau 8}

Présence aux rencontres de la CoP et participation aux activités proposées (phase 1)

\begin{tabular}{lccc}
\hline Participantes & $\begin{array}{c}\text { Présences aux } \\
\text { CoP } \\
(\mathrm{n}=5)\end{array}$ & $\begin{array}{c}\text { Activités } \\
\text { réalisées } \\
(\mathrm{n}=4)\end{array}$ & $\begin{array}{c}\text { Activités } \\
\text { déposées } \\
(\mathrm{n}=4)\end{array}$ \\
\hline Enseignante 1 & 5 & 4 & 3 \\
Enseignante 2 & 4 & 3 & 2 \\
Enseignante 3 & 4 & 3 & 3 \\
Enseignante 4 & 5 & 3 & 3 \\
Enseignante 5 & 3 & 2 & 1 \\
Enseignante 6 & 3 & 4 & 4 \\
\hline
\end{tabular}

À partir de ces tableaux, nous pouvons constater que la présence et l'investissement dans les activités de la CoP varient effectivement entre les participantes. Certaines ont une participation régulière et constante de même qu'un investissement réel dans les activités. Tandis que d'autres ont une participation plus irrégulière et un investissement asymétrique dans les activités. Ainsi, en nous basant sur le modèle de Wenger, McDermott et Snyder (2002), nous pouvons effectivement dégager trois niveaux de participation, soit l'entrepreneur, le participant et le témoin.

Pour dégager les profils de participantes présentes dans notre $\mathrm{CoP}$, nous avons croisé les niveaux de participation obtenus avec la fréquence et à la qualité des interventions des participantes au regard de l'avancement du discours collectif, soit naïf, novice, apprenti et expert. Ainsi, il en résulte 12 profils potentiels dans lesquels nous avons réparti les participantes en fonction de leurs interactions respectives. Le Tableau 9 présente ces profils et la Figure 2, la répartition des participantes dans ces profils, laquelle est basée sur le modèle de participation de Wenger, McDermott et Snyder (2002). Sans surprise, la participante E1 se retrouve dans le profil Expert entrepreneur. 


\section{REVUE HYBRIDE DE L'ÉDUCATION}

\section{Tableau 9}

Profils de participantes de la CoP

\begin{tabular}{|c|c|c|}
\hline $\begin{array}{c}\text { Niveaux } \\
\text { d'expertise }\end{array}$ & $\begin{array}{c}\text { Profil de } \\
\text { participation }\end{array}$ & Description du profil \\
\hline \multirow[t]{3}{*}{ Naïf } & Entrepreneur & $\begin{array}{l}\text { La présence est constante et régulière. Le } \\
\text { membre s'investit dans chacune des } \\
\text { activités de la communauté de pratique et } \\
\text { participe activement lors des rencontres; } \\
\text { interventions fréquentes et soutenues, } \\
\text { mais plusieurs sont de niveau naîf faisant } \\
\text { peu progresser les interactions et la } \\
\text { discussion dans la CoP. }\end{array}$ \\
\hline & Participant & $\begin{array}{l}\text { La présence est plus au moins constante et } \\
\text { régulière. Le membre s'investit dans } \\
\text { certaines activités et participe de façon } \\
\text { active aux rencontres auxquelles il est } \\
\text { présent; interventions assez fréquentes et } \\
\text { soutenues, mais plusieurs sont de niveau } \\
\text { naïf faisant peu progresser les interactions } \\
\text { et la discussion dans la CoP. }\end{array}$ \\
\hline & Témoin & $\begin{array}{l}\text { La présence n'est pas constante et } \\
\text { régulière. Le membre s'investit peu dans } \\
\text { les activités et participe de façon } \\
\text { périphérique aux rencontres auxquelles il } \\
\text { est présent. Ses interventions sont peu } \\
\text { fréquentes et soutenues, mais plusieurs } \\
\text { sont de niveau naïf faisant peu progresser } \\
\text { les interactions et la discussion dans la } \\
\text { CoP. }\end{array}$ \\
\hline \multirow[t]{2}{*}{ Novice } & Entrepreneur & $\begin{array}{l}\text { La présence est constante et régulière. Le } \\
\text { membre s'investit dans chacune des } \\
\text { activités de la communauté de pratique et } \\
\text { participe activement lors des rencontres; } \\
\text { interventions fréquentes et soutenues; } \\
\text { plusieurs sont de niveau novice ou apprenti } \\
\text { faisant peu progresser les interactions et la } \\
\text { discussion dans la CoP. }\end{array}$ \\
\hline & Participant & $\begin{array}{l}\text { La présence est plus au moins constante et } \\
\text { régulière. Le membre s'investit dans } \\
\text { certaines activités et participe de façon } \\
\text { active aux rencontres auxquelles il est } \\
\text { présent; interventions assez fréquentes et } \\
\text { soutenues; plusieurs sont de niveau } \\
\text { novice ou apprenti faisant peu progresser }\end{array}$ \\
\hline
\end{tabular}




\section{REVUE HYBRIDE DE L'ÉDUCATION}

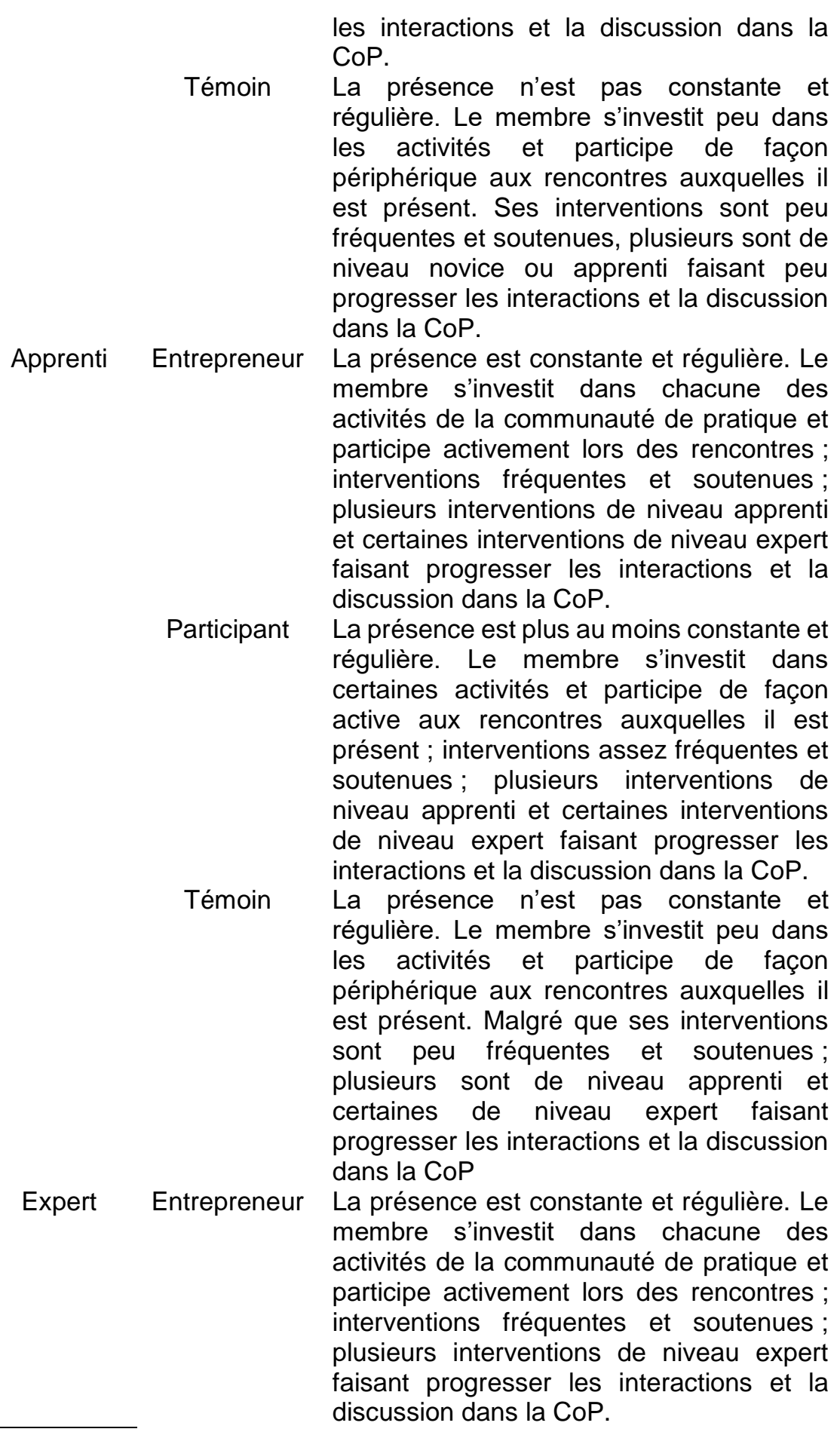




\section{REVUE HYBRIDE DE L'ÉDUCATION}

Participant La présence est plus au moins constante et régulière. Le membre s'investit dans certaines activités et participe de façon active aux rencontres auxquelles il est présent ; interventions assez fréquentes et soutenues; plusieurs interventions de niveau expert faisant progresser les interactions et la discussion dans la CoP

Témoin La présence n'est pas constante et régulière. Le membre s'investit peu dans les activités et participe de façon périphérique aux rencontres auxquelles il est présent. Malgré que ses interventions sont peu fréquentes et soutenues, plusieurs sont de niveau expert faisant progresser les interactions et la discussion dans la CoP

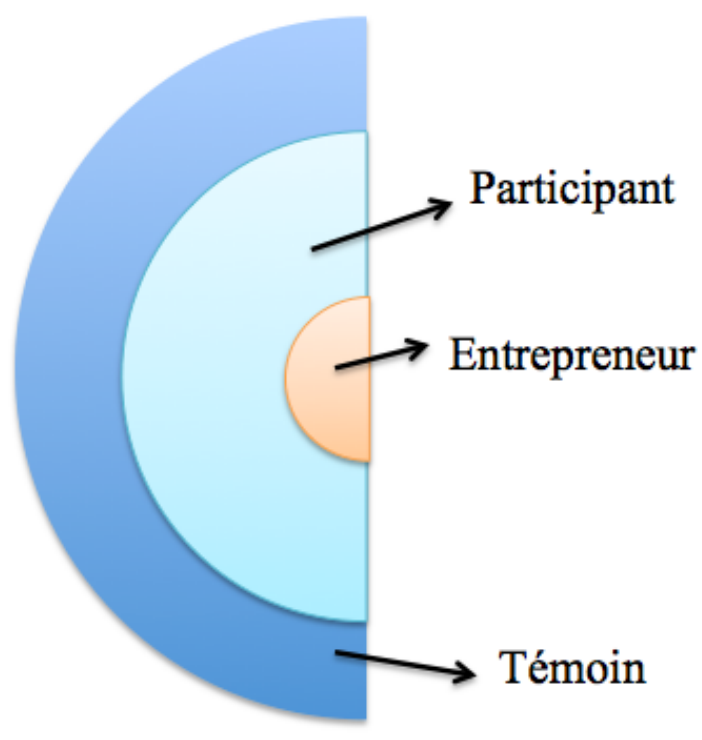

- Expert : E5 phase 2

- Apprenti: E4 et E10

- Novice: E2 et E3

- Naïf : (aucune)

- Expert : E1 phases 1 et 2

- Apprenti : E11 et E12

- Novice : (aucune)

- Naïf : (aucune)

- Expert : (aucune)

- Apprenti : E13

- Novice: E6

- Naï: E5 phase 1

Figure 2. La répartition des participantes dans les profils de participation potentiels

La participante E5 est quant à elle dans deux profils différents en fonction de la phase de la CoP ; lors de la phase 1 elle était dans le profil Naïf témoin et lors de la phase 2 dans le profil Expert participant. De plus, de façon générale, les participantes de la phase 2 sont réparties entre les 


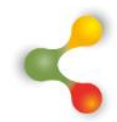

\section{REVUE HYBRIDE DE L'ÉDUCATION}

profils Expert et Apprenti tandis que certaines participantes de la phase 1 sont soit dans les profils Novice ou Naïf.

Finalement, au-delà des données présentées jusqu'à maintenant, il est à noter que les données sociodémographiques présentées dans la section méthodologie ne semblent pas présenter de liens potentiels quant à la répartition des participantes dans les profils de participation centraux. En effet, il y a une très grande variabilité entre les participantes du projet du point de vue de l'âge, de l'expérience d'enseignement et de la région de provenance comme en témoignent les pourcentages et les écarts types présentés dans le tableau 1. Or, au sein de chaque profil, cette même variabilité demeure. À titre d'exemple, les enseignantes E1, E5, E11 et E12 proviennent de quatre régions différentes. Elles enseignent dans des classes qui sont soit multicycles, de premier cycle, de deuxième cycle ou de troisième cycle. Elles représentent les tranches d'âges « 35-39 », « 4044 », «50-54 », «55-59 » et leur nombre d'années d'expérience d'enseignement varie entre 13 (min.) et 30 (max.).

\section{Discussion}

En conclusion, pour répondre à notre première question, les interactions ont effectivement évolué entre la première et la deuxième phase de la CoP. Les participantes sont intervenues plus fréquemment, plus longuement, et ont fait plus souvent des interventions visant l'avancement du discours collectif que lors de la première phase. Ainsi, nous pouvons constater que selon les stades de développement d'une CoP (Langelier, 2005 ; Wenger et al., 2002) présentés précédemment, lors de la première phase, la CoP semblait encore en période de démarrage et, après son unification, l'étape de maturité fut atteinte lors de la deuxième phase pour mener à l'avancement du discours collectif. La participante E5 est d'ailleurs un bon indicateur de cette évolution, comme elle a participé aux deux phases et a considérablement augmenté la fréquence et la qualité de ses interventions quant à la prise en compte des autres membres dans le discours collectif lors de sa deuxième année de participation. À cet effet, il semble que les changements organisationnels apportés lors de la deuxième phase ont contribué à l'évolution observée. Ainsi, le fait d'augmenter le nombre de rencontres, passant de cinq à huit, semble avoir permis de laisser plus de temps pour mieux faire avancer la communauté de pratique. Ce résultat est d'ailleurs cohérent avec l'un des critères d'efficacité du développement professionnel établis par Wood et Stanulis (2009), soit que le dispositif doit « être suffisamment long et fréquent pour assurer une acquisition progressive de connaissances et de confiance chez les participants " et Desimone, 2009. Toutefois, comme nous avons constaté qu'au même nombre de rencontres, soit lors des cinq premières des deux phases, les interactions étaient déjà différentes, il semble qu'audelà du nombre de rencontres, le changement d'animation, attribué à une étudiante plutôt qu'à la chercheuse principale, pourrait aussi avoir eu un impact sur les interactions à l'intérieur de la communauté de pratique. Ainsi, 


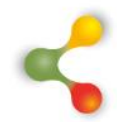

\section{REVUE HYBRIDE DE L'ÉDUCATION}

les participantes ont peut-être été plus en mesure d'interagir spontanément avec une animatrice qui partageait une identité commune avec elles de même qu'un répertoire de pratiques commun. D'ailleurs, lors de la deuxième rencontre de la phase 2 , soit la rencontre à laquelle la chercheuse principale a participé activement, la fréquence des interactions des participantes est considérablement plus basse que lors des autres rencontres. Comme cette rencontre portait davantage sur l'acquisition de connaissances théoriques au sujet du langage, cela peut avoir influencé les interactions. Par conséquent, il semble que l'animation de la CoP par un ou une spécialiste pourrait avoir un impact non négligeable sur les interactions et la responsabilisation des membres par rapport au discours collectif dans la communauté de pratique. II s'agit donc d'un résultat prometteur quant à la responsabilisation et la prise en charge autonome de la communauté de pratique (Boutin et Hamel, 2017), c'est-à-dire l'accès à des connaissances fines au moment requis à l'aide d'experts du domaine. Ce résultat n'est pas sans faire écho aux facteurs organisationnels liés au succès des CoPV proposés par Bourhis et ses collaborateurs (2005). Rappelons que leurs travaux portant sur 18 CoPV ont démontré que l'environnement organisationnel, notamment, était étroitement lié à leur succès. De plus, la structure de l'équipe de coordination semble aussi avoir un impact non négligeable puisque parmi les CoPV qu'ils ont identifié comme ayant échoué, l'une d'entre elles a fait face à un problème similaire, c'est-à-dire que l'animateur était un expert de contenu, mais avait de la difficulté à établir des liens avec les membres de la CoPV (Dubé, Bourhis et Jacob, 2005).

Pour ce qui est de notre deuxième question de recherche, il se dégage effectivement des profils de participation à l'intérieur de la communauté de pratique en fonction des interactions et de la participation, lesquels sont cohérents avec les niveaux de participation de Wenger, McDermott et Snyder (2002). Toutefois, il nous apparait prometteur de croiser à ces niveaux la qualité des interventions des membres de la CoP au regard de la prise en compte des autres membres dans la négociation de sens afin de bien rendre compte de leur responsabilisation face à l'avancement du discours collectif. Plusieurs auteurs se sont d'ailleurs intéressés à cet aspect dans le contexte du discours écrit, à l'intérieur de forum collaboratif par exemple (Bereiter et Scardamalia, 1987 ; Boutin, Hamel et Laferrière, 2016; Garrison et al., 2000; Murphy, 2004; Scardamalia et Bereiter, 2003). À cet effet, il est intéressant de remarquer que les participantes de la deuxième phase sont plus portées à faire des interventions qui permettent cet avancement que celles de la première phase. D'ailleurs, il importe de mentionner à ce sujet que les participantes E11 et E12, qui étaient dans le profil Apprenti entrepreneur, ont décidé de poursuivre la communauté de pratique au sein de l'ÉER pour une troisième phase et de s'impliquer au sein de l'équipe de coordination. Elles ont donc pris la responsabilité de la CoP et de sa pérennisation, ce qui est pourtant difficile à réaliser comme l'ont souligné Boutin et Hamel (2017). Ainsi, malgré la fin du projet et donc l'atteinte inévitable de l'étape du momentum, 


\section{$\&$}

\section{REVUE HYBRIDE DE L'ÉDUCATION}

la CoP se transforme au lieu de se dissoudre (Langelier, 2005 ; Wenger et al., 2002). II semble donc qu'il faut miser sur la responsabilisation des membres face à l'avancement du discours collectif pour rendre la communauté pérenne (Dameron et Josserand, 2007 ; McDermott, 2000 ; Wenger, McDermott et Snyder, 2002) et donc, leur permettre d'accéder aux profils d'entrepreneurs, notamment en leur accordant suffisamment de temps et d'espace afin qu'ils se sentent en confiance et légitimes dans le discours (Wenger, McDermott et Snyder, 2002 ; Wood et Stanulis, 2009). L'attribution de la tâche d'animation est alors un aspect à ne pas négliger dans la mise en place d'une CoP, car si c'est une personne experte qui s'en charge, nos résultats, tout comme ceux de Dubé et al. (2005) semblent démontrer que les autres membres de la communauté soient alors moins enclins à prendre leur place et à se sentir en confiance.

Certaines limites sont à considérer dans l'interprétation de ces résultats. D'abord, le fait qu'il fut difficile d'obtenir un alpha d'accord interjuge élevé peut remettre en question le codage effectué. Par contre, comme il s'agit d'un nombre élevé de codes, il n'est pas surprenant qu'un alpha d'accord au-delà de $80 \%$ soit plus difficile à obtenir (Krippendorff, 2013). De plus, il aurait été intéressant de confronter les participantes aux données dans une approche plus centrée sur l'activité réelle, où celles-ci auraient été amenées à décrire leur propre vécu dans la communauté de pratique. Cette démarche aurait certainement permis de confirmer davantage leurs propres perceptions de leur participation et de leurs interactions au sein de la CoP (Lussi Borer, Durand et Yvon, 2015 ; Poizat, Salini et Durand, 2013 ; Theureau, 2004). Finalement, il serait intéressant de documenter la poursuite de la CoP en dehors du projet, laquelle sera prise en charge par deux participantes dans l'ÉER. En effet, il s'agit d'une avenue qui ne semble pas avoir été explorée pour le moment, malgré l'importance de ces données pour bien comprendre comment arriver à rendre les communautés de pratique pérennes une fois que les projets de recherche les supportant se terminent (Dameron et Josserand, 2007 ; McDermott, 2000 ; Wenger, McDermott et Snyder, 2002). 


\section{REVUE HYBRIDE DE L'ÉDUCATION}

\section{Références}

Bereiter, C. et Scardamalia, M. (1987). The Psychology of Written Composition. Lawrence Erlbaum Associates. http://www.ikit.org/fulltext/1987thepsychology/ Preface.pdf

Bissonnette, S. et Richard, M. (2010). Les modalités d'efficacité de la formation continue. Vivre le primaire, 23(3), 34-36.

Blank, R. K et De Las Alas, N. (2009). Effects of teacher professional development on gains in student achievement. How meta analysis provides scientific evidence useful to education leaders. Council of Chief State School Officers. https://web.b.ebscohost.com/ehost/detail/detail?vid=3\&sid=1a42942c -558c-46cc-b6fb-f35b450b7943\%40pdc-vsessmgr01\&bdata $=$ JmxvZ2luLmFzcCZsYW5nPWZyJnNpdGU9ZWh vc3QtbG|2ZQ\%3d\%3d\#AN=ED514190\&db=eric

Boix-Mansilla, V. et Gardner, H. (1998). What are the Qualities of Understanding. Dans Teaching for understanding: Linking research with practice (p.161-196). Jossey-Bass Publishers.

Bourhis, A. et Tremblay, D. G. (2004). Les facteurs organisationnels de succès des communautés de pratique virtuelles. Collection Recherche et études de cas.

Boutin, P.-A., Hamel, C. et Laferrière, T. (2016). La sélection d'idées prometteuses et l'émergence d'un questionnement authentique dans l'élaboration du discours collectif d'élèves du primaire. La Revue canadienne de l'apprentissage et de la technologie, 42(1), 1-23. https://www.cjlt.ca/index.php/cj|t/article/view/27455/20207

Boutin, P.-A. et Hamel, C. (2017). La participation à l'élaboration d'un discours collectif asynchrone comme soutien au développement professionnel des enseignants québécois faisant partie de l'équipe d'accompagnement de l'École en réseau. Distances et médiations des savoirs, (17). doi.org/10.4000/dms.1763

Braze, D., Tabor, W., Shankweiler, D.P. et Mencl, W.E. (2007). Speaking up for vocabulary: Reading skill differences in young adults. Journal of Learning Disabilities, 40(3), 226-243.

Cain, K. et Oakhill, J. (dir.). (2008). Children's comprehension problems in oral and written language : A cognitive perspective. Guifford Press.

Catts, H.W., Fey, M.E., Tomblin, J.B. et Zhang, X. (2002). A longitudinal investigation of reading outcomes in children with language 


\section{REVUE HYBRIDE DE L'ÉDUCATION}

impairments. Journal of Speech, Language, and Hearing Research, 45, 1142-1157. doi:10.1044/1092-4388 (2002/093)

Dameron, S. et Josserand, E. (2007). Le développement d'une communauté de pratique. Une analyse relationnelle. Revue française de gestion, 33(174), 131-148. doi.org/10.3166/rfg.174.131-148

Desimone, L.M. (2009). Improving impact studies of teachers' professional development: Toward better conceptualizations and measures. Educational researcher, 38(3), 181-199.

Dubé, L., Bourhis, A. et Jacob, R. (2005). The impact of structuring characteristics on the launching of virtual communities of practice. Journal of Organizational Change Management, 18(2), 145166.

Garrison, D.R., Anderson, T. et Archer, W. (2000). Critical inquiry in a textbased environment: computer conferencing in higher education. The Internet and Higher Education, 2(2), 87-105.

Gaudreau, N. et Nadeau, M.-F. (2015). Enseigner aux élèves présentant des difficultés comportementales: dispositifs pour favoriser le développement des compétences des enseignants. La nouvelle revue de l'adaptation et de la scolarisation, 4(72), 27-45.

Hammer, C.S., Morgan, P., Farkas, G., Hillemeier, M., Bitetti, D. et Maczuga, S. (2017). Late talkers: A population-based study of risk factors and school readiness consequences. Journal of Speech, Language, and Hearing Research, 60(3), 607-626.

Hattie, J. (2009). Visible learning: a synthesis of over 800 meta-analyses relating to achievement. Routledge.

Krippendorff, K. (2013). Content Analysis. An Introduction to Its Methodology ( $3^{e}$ éd.). Sage Publications.

Langelier, L. (2005). Guide de mise en place et d'animation de Communautés de pratique intentionnelles : travailler, apprendre et collaborer en réseau. Centre Francophone de Recherche en Informatisation des Organisations [CEFRIO]. http://www.cefrio.qc.ca/media/uploader/travailler_apprendre_collab orer.pdf

Lussi Borer, V., Durand, M. et Yvon, F. (2015). Analyser le travail pour former les professionnels de l'éducation? Dans V. Lussi Borer, M. Durand et F. Yvon (dir.), Analyse du travail et formation dans les métiers de l'éducation (p. 7-29). De Boeck Supérieur. 


\section{$\&$}

\section{REVUE HYBRIDE DE L'ÉDUCATION}

Lussi Borer, V. et Muller, A. (2017). Designing collaborative video learning lab to transform teachers's work practices. Dans P.G. Rossi et L. Fedeli (dir.), Integrating video into pre-service and in-service teacher training (p. 68-89). IGI Global.

McDermott, R. (2000). Knowing in Community?: 10 Critical Success Factors in Building Communities of Practice. IHRIM Journal, 3, 1-12. http://www.a-i-a.com/capital-intelectual/KnowinglnCommunity.pdf

Miles, M. B., et Huberman, M. A. (2003). Analyse des données qualitatives. De Boeck.

Ministère de l'Éducation, du Loisir et du Sport (MELS). (2006). Programme de formation de l'école québécoise: éducation préscolaire et enseignement primaire. Gouvernement du Québec.

Murphy, E. (2004). Recognising and Promoting Collaboration in an Online Asynchronous Discussion. British Journal of Educational Technology, 35(4), 421-431.

Nation, K., Clarke, P., Marshall, C.M. et Durand, M. (2004). Hidden language impairments in children: Parallels between poor reading comprehension and specific language impairment? Journal of speech, language and hearing research, 47(1), 199-211. doi :10.1044/1092-4388(2004/017)

National Institute of Child Health and Human Development (NICHID) Early Child Care Research Network. (2005). Pathways to reading: The role of oral language in the transition to reading. Developmental Psychology, 41(2), 428-442. doi:10.1037/0012-1649.41.2.428

Oakhill, J.V. et Cain, K. (2004). The development of comprehension skill. Dans T. Nunes et P. Bryant (dir.), Handbook of children's literacy ( $p$. 155-180). Kluwer Academic.

Piasta, S.B., Connor, M.C., Fishman, B.J. et Morrison, F.J. (2009). Teachers' knowledge of literacy concepts, classroom practices, and student reading growth. Scientific Studies of Reading, 13(3), 224-248.

Poizat, G., Salini, D. et Durand, M. (2013). Approche énactive de l'activité humaine, simplexité et conception de formations professionnelles. Education Sciences et Society, 4(1), 97-112.

Scarborough, H. (2001). Connecting early language and literacy tolater reading dis(abilities): Evidence, theory and practice. Dans S. Neuman et D. Dickinson (dir.), Handbook of early literacy research (p. 97-109). Guilford Press. 


\section{REVUE HYBRIDE DE L'ÉDUCATION}

Scardamalia, M. (2002). Collective cognitive responsibility for the advancement of knowledge. Dans B. Smith (dir.), Liberal education in a knowledge society (p.67-98). Open Court. http://ikit.org/fulltext/2002CollectiveCog.pdf

Scardamalia, M. et Bereiter, C. (2003). Knowledge building. Dans J.W. Guthrie (dir.), Encyclopedia of education. ( $2^{\mathrm{e}}$ éd., p. 13701373).Macmillan. http://ikit.org/fulltext/inpressKB.pdf

Schatschneider, C., Fletcher, J.M., Francis, D.J., Carlson, C.D. et Foorman, B. R. (2004). Kindergarten prediction of reading skills: A longitudinal comparative analysis. Journal of Educational Psychology, 96(2), 265282. doi:10.1037/0022-0663.96.2.265

Snoeck, C. et Tilman, C. (2014, juillet). Communautés de pratique : production et partage de connaissances dans le monde hospitalier [communication orale]. Gestion et Ingénierie des Systèmes Hospitaliers (GISEH), Liège, Belgique. https://orbi.uliege.be/handle/2268/179088

Storch, S.A. et Whitehurst, G.J. (2002). Oral language and coderelated precursors to reading: Evidence from a longitudinal structural model. Developmental Psychology, 38(6), 934-947. doi:10.1037/00121649.38.6.934

St-Pierre, M.-C., Bélisle, B., Hamel, C., Desmarais, C., Brissette, C., StOnge, J. et Laferrière, T. (2015, août). Compréhension de lecture et habiletés inférentielles: exploration des connaissances et des pratiques d'enseignants au primaire. $2^{\mathrm{e}}$ symposium sur la littératie à l'école [symposium]. Sherbrooke, Canada.

Theureau, J. (2006). Le cours d'action: Méthode développée. Octares Éditions.

Verhoeven, L. et Van Leeuwe, J. (2008). Prediction of the development of reading comprehension: A longitudinal study. Applied Cognitive Psychology 22(3), 407-423. doi:10.1002/acp.1414

Wenger, E. (2005). La théorie des communautés de pratique. Presses de I'Université Laval.

Wenger, E., McDermott, R. et Snyder, W.M. (2002). Communities of Practice and their Value to Organizations. Cultivating Communities of Practice. Havard Business School Press, 1-47. doi.org/10.1016/j.jchas.2013.03.426 


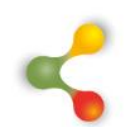

\section{REVUE HYBRIDE DE L'ÉDUCATION}

Wood, A. L. et Stanulis, R. N. (2009). Quality Teacher Induction: " FourthWave " (1997-2006) Induction Programs. The New Educator, 5(1), 1 23. doi:10.1080/1547688X.2009.10399561 